\title{
PERFIL DE PACIENTES HEMODIALÍTICOS RELACIONADOS A PARÂMETROS BIOQUÍMICOS E HEMATOLÓGICOS NO OESTE CATARINENSE
}

\author{
Elisangela Giachini, Camila Zanesco, Silvia Silva de Souza, Paôla Cristina Ceratto, Débora Tavares de \\ Resende e Silva
}

Universidade Federal da Fronteira Sul-UFFS. E-mail: eli_giachini94@hotmail.com

\begin{abstract}
RESUMO
Este estudo teve o objetivo de analisar a adequação sanitária de um serviço de hemodiálise (HD) no oeste catarinense, no decorrer de 2014, relacionando-a aos parâmetros bioquímicos, hematológicos apresentados pelos pacientes hemodialítico e o trabalho da equipe de enfermagem. Pesquisa de caráter observacional, exploratória, transversal com amostragem sistemática aleatória. Foram encontradas alterações nos parâmetros bioquímicos e hematológicos referentes ao Grupo 1 (3-7 meses de HD) em relação a outros três Grupos (8-12, 13-24 e 25-36 meses de HD). Alterações nos parâmetros bioquímicos e hematológicos estão presentes em pacientes hemodialíticos, especialmente no que se refere aos biomarcadores inflamatórios, refletindo maior fragilidade imunológica. Tais parâmetros são ferramentas para avaliar a qualidade e efetividade da terapia de hemodiálise, assim como o acompanhamento do enfermeiro durante as seções de hemodiálise na evolução clínica do paciente.
\end{abstract}

Palavras-chave: rim, hemodiálise, doença renal crônica, vigilância sanitária.

\section{PROFILE OF PATIENTS WITH HEMODIALYSIS RELATED TO BIOCHEMICAL AND HEMATOLOGICAL PARAMETERS IN THE WEST OF SANTA CATARINA}

\begin{abstract}
This study aimed to analyze the health suitability of a hemodialysis service (HD) in western Santa Catarina, during 2014, relating to the biochemical and hematological parameters presented by haemodialysis patients and the work of the nursing staff. It was an observational, exploratory, cross-sectional research, with random systematic sampling. It was found change in biochemical and hematological parameters, the group 1 (3-7 months of HD) regarding others three groups (8-12, 13-24 e 25-36 months of HD). The hemodialysis service found to comply with the sanitary specifications and period the nursing team proved helpful in care for the patients. Changes in biochemical and hematological parameters were present in hemodialysis patients, especially in refers to inflammatory biomarkers, reflecting the greater immune weakness. Such parameters are tools to assess the quality and effectiveness of therapy hemodialysis, as well as the monitoring of the nurse during hemodialysis sessions in the clinical evolution of the patient.

Keywords: kidney, hemodialysis, chronic kidney disease, health surveillance.
\end{abstract}

\section{INTRODUÇÃO}

Nas últimas décadas houve uma melhora significativa das condições de saúde no país, gerando um impacto sobre a estrutura etária. A redução da fração jovem da população e a ampliação da população idosa implicam em um novo perfil epidemiológico e de morbidade e mortalidade, caracterizando um período de transição, onde doenças infecto-parasitárias coexistem com a elevação da prevalência de Doenças Crônicas Não-Transmissíveis (DCNT) $)^{1,2,3}$.
Dentre esse conjunto de doenças denominadas crônicas não-transmissíveis, encontra-se a Doença Renal Crônica (DRC), considerada a nova epidemia do século XXI, uma síndrome clínica que caracteriza-se pela redução significativa, lenta, gradual e progressiva das funções renais excretoras, endócrinas e metabólicas. Sua incidência vem aumentando e apresenta elevados índices de morbimortalidade mesmo na fase pré-dialítica ${ }^{4,5}$. 
A DRC afeta o metabolismo hídrico, eletrolítico e acidobásico, além de repercutir em alterações no metabolismo dos macronutrientes, propiciando situações de hipercatabolismo, próinflamatórias e pró-oxidativas ${ }^{5}$. Estudos recentes indicam que estes desfechos indesejados podem ser prevenidos ou retardados se esta for diagnosticada e tratada precocemente. Infelizmente, na maior parte dos casos, a DRC é subdiagnosticada e tratada de forma inadequada devido à dificuldade do diagnóstico e falta de investigações mais aprofundadas.

A disfunção renal quase sempre apresenta evolução progressiva, insidiosa e assintomática, a qual resulta em complicações decorrentes da perda funcional renal e óbito, principalmente por causas cardiovasculares ${ }^{6,7}$. Casos avançados da DRC necessitam de intervenções mais invasivas como a Terapia Renal Substitutiva (TRS), dentre as modalidades, a mais usual é a Hemodiálise (HD) ${ }^{8,9}$. A HD em alguns casos pode gerar insatisfação e limitação ao paciente. A equipe de enfermagem é de suma importância no tratamento junto à pacientes em tratamento de TRS, hemodiálise. Frente à relevância dessa doença crônica, podemos destacar as atribuições e competências do enfermeiro no atendimento aos pacientes por meio da abordagem dos fatores de risco, desenvolvimento de atividades educativas, interação, cuidado e assistência, assim como a promoção de saúde e controle de complicações. Uma das medidas de controle destas complicações pode ser efetuada pelo cumprimento da Resolução no 154 de 15 de Junho de 2004, a qual define além de outras tantas, a obrigatoriedade do serviço de diálise realizar periodicamente, em seus pacientes os exames mensais, trimestrais, semestrais e anuais. Contar com o profissional enfermeiro no serviço é de extrema importância para a orientação dos pacientes, enfatizando a importância do cumprimento do tratamento. A equipe de enfermagem é a que tem maior proximidade com o paciente no serviço, desta forma os profissionais devem estar atentos a qualquer ocorrência que possa surgir, gerando uma resposta precoce impedindo muitas vezes maiores complicações que possam vir a acontecer ${ }^{10,11}$.

Dessa forma este estudo teve como objetivo verificar o perfil clínico dos usuários do serviço de hemodiálise em questão, qual grupo de pacientes apresenta o perfil bioquímico e hematológico com menores anormalidades e qual é o papel do enfermeiro no cuidado do paciente renal crônico.

\section{METODOLOGIA}

O estudo foi de caráter observacional, exploratória, transversal com análise quantitativa analítica. Foi realizada em uma Clínica Renal da região oeste de Santa Catarina, localizada no município de Chapecó contemplando uma amostragem sistemática aleatória. Teve como população-alvo indivíduos de ambos os sexos que realizavam Terapia Renal Substitutiva (TRS) na modalidade de Hemodiálise (HD) no mês de agosto de 2014, com um período de tratamento maior que três meses e menor que 36 meses. Sendo excluídos da amostra pacientes que foram a óbito e aqueles que realizaram Transplante Renal (TR) durante a pesquisa.

A coleta de dados foi realizada no período de Agosto a Novembro de 2014, sendo iniciada a coleta somente após aprovação do projeto no Comitê de Ética em Pesquisa da Universidade Federal da Fronteira Sul (№ 33713514.7.0000.5564) e assinatura do Termo de Consentimento Livre e Esclarecido (TCLE) pelos pacientes.

Durante o período destinado a coleta de dados no ambiente do serviço, buscou-se observar qual o papel do profissional de enfermagem no serviço e sua atuação junto ao paciente em tratamento hemodialítico.

Participantes

Primeiramente foi realizado o levantamento dos indivíduos que se encaixavam na amostra de acordo com os critérios de inclusão e exclusão, resultando em 77 pacientes. Destes, um foi excluído por ter realizado TR, seis por terem ido a óbito e 20 por não assinarem o TCLE, restando 50 pacientes. Os mesmos foram separados em quatro grupos, conforme o tempo de tratamento de HD. Os pacientes foram agrupados conforme o tempo em HD sendo:

- Grupo 1 entre 3-07 meses em HD, n=11 pacientes (22\%);

- Grupo 2 entre 8-12 meses em HD, n=15 pacientes (30\%);

- Grupo 3 entre 13-24 meses em HD, $\mathrm{n}=12$ pacientes $(24 \%)$;

- Grupo 4 entre 25-36 meses em HD, $\mathrm{n}=12$ pacientes $(24 \%)$.

Os dados foram obtidos a partir do prontuário eletrônico de cada paciente e transcritos em planilhas para posterior análise. 
Foram coletadas informações quanto ao tempo (meses) de tratamento de HD na vida, gênero, etnia, idade, peso, altura e posteriormente realizado o cálculo do Índice de Massa Corporal (IMC).

Parâmetros hematológicos e bioquímicos

Exames Mensais: foram coletadas amostras para dosagem de hemoglobina total ou hematócrito (HT), hemoglibina (HB), ureia pré e pós-sessão de $\mathrm{HD}, \mathrm{K}^{+}, \mathrm{Ca}^{2+}, \mathrm{PO}_{3}{ }^{2-}$, transaminase glutâmico pirúvica (TGP), creatinina (CR) e Proteína C Reativa (PCR), dos últimos oito meses, o que inclui os meses de março a outubro.

Exames trimestrais: foram realizados exames de Saturação da Transferrina, Dosagem de Ferritina, Ferro Sérico, Proteínas Totais, Albumina e Fosfatase Alcalina. Foram coletados os dados referentes a estes meses, obtendo-se resultados dos exames realizados no 2 ㅇ, 3 e e 4 은 trimestre do ano de 2014.

Exame semestral: foram realizados exames de Paratormônio (PTH), sendo coletado o resultado do 1ㅇ e 2으 semestre de 2014.

Exames anuais: Nível sérico de alumínio $\left(\mathrm{Al}^{3+}\right)$, Colesterol LDL, Colesterol HDL, Colesterol VLDL, Triglicerídeos, dosagem de anticorpos para HIV. Foram coletados os resultados disponíveis no prontuário que datasse do ano de 2014.

Em paralelo, realizou-se a observação geral da equipe de enfermagem no local.

Análise estatística

A normalidade foi testada pelo teste de Kolmogorov-Smirnov. As diferenças entre os grupos, em relação às variáveis do estudo, foram avaliadas por meio da análise de variância oneway ANOVA. Quando as amostras não apresentaram distribuição normal, foram submetidas à análise de variância, pelo teste de Kruskal-Wallis. Quando houve diferença, os grupos foram comparados entre si com correção de Student-Newman-Keuls para as variáveis paramétricas e correção pelo teste de Dunn para variáveis não paramétricas. Os resultados foram apresentados como média aritmética dos valores e desvio padrão (DP) para variáveis paramétricas, e sob a forma de mediana e faixa de variação para variáveis não paramétricas. Foram consideradas estatisticamente significantes as diferenças em que a probabilidade de rejeição da hipótese de nulidade foi menor que $p<0,05$.

\section{RESULTADOS} pacientes

Caracterização epidemiológica dos

Dos 50 pacientes, 30 (60\%) eram do gênero masculino, todos de etnia caucasiana, nenhum possuía hepatite $B$ ou $C$ e todos eram HIV negativos. O percentual de pacientes em HD com idade entre 19 a 64 anos, 65 a 80 anos e acima de 80 anos foi de $56 \%(n=28), 32 \%(n=16)$ e $1,2 \%(n=6)$, respectivamente. Com idade mínima de 22 anos e máxima de 95 anos. Em relação ao IMC, o grupo 1 apresentou uma média no limite da normalidade, enquanto os grupos 2,3 e 4 apresentaram $I M C \geq 25 \mathrm{Kg} / \mathrm{m}^{2}$, indicando sobrepeso (Tabela 1).

Tabela 1. Características epidemiológicas dos participantes.

\begin{tabular}{lccccc}
\hline & Grupo 1 & Grupo 2 & Grupo 3 & Grupo 4 & p \\
\hline Idade (anos) & $59,00 \pm 16,93$ & $63,73 \pm 16,54$ & $61,67 \pm 14,27$ & $62,75 \pm 18,87$ & 0,9599 \\
\hline Peso $(\mathrm{Kg})$ & $65,55 \pm 16,76$ & $66,80 \pm 12,94$ & $71,08 \pm 15,97$ & $68,25 \pm 17,62$ & 0,7941 \\
\hline Estatura $(\mathrm{cm})$ & $162,6 \pm 9,08$ & $163,3 \pm 8,29$ & $163,4 \pm 10,81$ & $161,3 \pm 10,25$ & 0,9589 \\
\hline IMC $\left(\mathrm{Kg} / \mathrm{m}^{2}\right)$ & $24,71 \pm 5,47$ & $25,07 \pm 4,81$ & $26,63 \pm 5,84$ & $26,05 \pm 5,43$ & 0,9479
\end{tabular}

Valores expressos em Média \pm DP; IMC = Índice de massa corporal.

Perfil bioquímico e hematológico dos pacientes

Exames mensais revelaram que os níveis de HB e HT estavam abaixo dos índices de normalidade nos quatro grupos, porém não foram encontradas diferença significativas nos resultados de HB, HT, ureia pós-diálise e TGP. Já as concentrações de TGP encontravam-se adequadas (Tabela 2). 
Tabela 2. Resultados dos exames mensais, trimestrais e semestrais.

Exames mensais

\begin{tabular}{|c|c|c|c|c|c|}
\hline & Grupo 1 & Grupo 2 & Grupo 3 & Grupo 4 & $\mathbf{p}$ \\
\hline HB (g/dL) & $10,02 \pm 0,57$ & $10,05 \pm 0,23$ & $10,20 \pm 0,31$ & $9,97 \pm 0,37$ & 0,5486 \\
\hline HT (\%) & $30,78 \pm 1,67$ & $31,20 \pm 0,69$ & $31,70 \pm 1,28$ & $31,13 \pm 1,03$ & 0,6988 \\
\hline Ureia pré-diálise $(\mathrm{mg} / \mathrm{dL})$ & $104,8 \pm 4,80$ & $125,6 \pm 7,89 *$ & $115,7 \pm 7,05$ & $119,0 \pm 10,23^{*}$ & 0,0007 \\
\hline Ureia pós-diálise $(\mathrm{mg} / \mathrm{dL})$ & $36,23 \pm 6,72$ & $41,15 \pm 2,54$ & $36,49 \pm 2,26$ & $40,12 \pm 4,00$ & 0,0762 \\
\hline Potássio $(\mathrm{mEq} / \mathrm{L})$ & $4,66 \pm 0,34$ & $5,359 \pm 0,19 *$ & $5,63 \pm 0,28 *$ & $5,30 \pm 0,21^{*}$ & 0,0003 \\
\hline Cálcio (mg/dL) & $8,66 \pm 0,26$ & $8,914 \pm 0,26$ & $9,14 \pm 0,36^{*}$ & $9,12 \pm 0,35$ & 0,0238 \\
\hline Fosfato $(\mathrm{mg} / \mathrm{dL})$ & $5,21 \pm 0,28$ & $5,888 \pm 0,60 *$ & $5,86 \pm 0,38^{*}$ & $5,78 \pm 0,24^{*}$ & 0,0076 \\
\hline TGP (U/L) & $26,68 \pm 7,84$ & $23,63 \pm 4,49$ & $24,58 \pm 4,39$ & $25,33 \pm 5,73$ & 0,8887 \\
\hline CR (mg/dL) & $5,97 \pm 0,19$ & $7,746 \pm 0,28 *$ & $8,13 \pm 0,74^{*}$ & $7,37 \pm 1,98$ & 0,0017 \\
\hline PCR (mg/L) & $16,84 \pm 10,91$ & $20,10 \pm 8,17$ & $13,23 \pm 3,45^{*}$ & $27,15 \pm 10,43^{*}$ & 0,0138 \\
\hline \multicolumn{6}{|c|}{ Exames trimestrais } \\
\hline Transferrina $(\mu \mathrm{g} / \mathrm{dL})$ & $176,4 \pm 3,04$ & $170,7 \pm 4,42$ & $174,8 \pm 2,83$ & $170,6 \pm 5,47$ & 0,2276 \\
\hline Ferritina $(\mathrm{ng} / \mathrm{mL})$ & $350,0 \pm 75,87$ & $465,8 \pm 81,2$ & $326,2 \pm 83,48$ & $413,5 \pm 101,0$ & 0,2875 \\
\hline Ferro sérico $(\mu \mathrm{g} / \mathrm{dL})$ & $73,48 \pm 12,35$ & $73,65 \pm 4,19$ & $80,00 \pm 10,00$ & $80,42 \pm 7,48$ & 0,7152 \\
\hline Fosfatase Alcalina (U/L) & $83,25 \pm 8,22$ & $94,67 \pm 8,81$ & $87,14 \pm 3,92$ & $101,3 \pm 5,79$ & 0,0787 \\
\hline Proteína Total $(\mathrm{g} / \mathrm{dL})$ & $6,34 \pm 0,23$ & $6,69 \pm 0,31$ & $6,70 \pm 0,06$ & $6,79 \pm 0,07$ & 0,1349 \\
\hline Albumina $(\mathrm{g} / \mathrm{dL})$ & $3,56 \pm 0,16$ & $3,74 \pm 0,18$ & $3,97 \pm 0,10$ & $4,01 \pm 0,13$ & 0,0656 \\
\hline \multicolumn{6}{|c|}{ Exame semestral } \\
\hline PTH unidade $(\mathrm{pg} / \mathrm{mL})$ & $181,9 \pm 52,92$ & $242,9 \pm 105,1$ & $176,1 \pm 91,87$ & $174,8 \pm 52,79$ & 0,7610 \\
\hline
\end{tabular}

HB - hemoglobina, HT - hematócrito, TGP - transaminase glutâmico pirúvica, CR - creatinina urinária, PCR - proteína C reativa, PTH - paratormônio. Valores expressos em Média \pm DP, * diferença significativa em comparação ao Grupo 1.

A ureia pré-diálise encontrou-se com níveis significativamente elevados entre o Grupo 1 com o Grupo 2 e Grupo 4 ( $t=17,12 ; p<0,0007)$. Em relação às concentrações de $\mathrm{K}^{+} \mathrm{o}$ grupo 1 demonstrou adequado, enquanto os grupos 2, 3 e 4 estão com concentrações significativamente superiores. Portanto, houve diferença estatística do Grupo 1 em relação aos Grupos 2 e 3 ( $t=18,65$; $\mathrm{p}<0,0003)$. As concentrações de cálcio do Grupo 1 apresentaram-se diminuídos significativamente em relação ao Grupo 3 ( $t=9,454 ; p<0,0238)$. Aumentos significativos nas concentrações de potássio, CR e PCR foram encontrados em todos os grupos. O fosfato apresentou significância estatística entre o Grupo 1 com os Grupos 2, 3 e $4(t=11,93 ; p<0,0076)$. Nas concentrações de CR foi encontrada diferença significativa entre $\mathrm{O}$ grupo 1 com os Grupos 2 e 3 ( $t=15,14 ; p<0,0017)$. A PCR apresentou diferença significativa para o Grupo 3 em relação ao Grupo 4 ( $t=10,64$; $\mathrm{p}<0,0138$ ) (Tabela 2).

Nos exames trimestrais em que foram quantificados os níveis de transferrina, ferritina, ferro, fosfatase alcalina, proteína total e albumina não foram encontradas diferença significativa entre os grupos. Além disto, o exame semestral em que foi quantificado o nível de PTH dos pacientes também não se observou diferença significativa entre os grupos, estando todos com concentrações adequadas (Tabela 2).

Em contrapartida, os exames anuais (Tabela 3) revelaram diferenças significativas entre os grupos. O colesterol HDL apresentou-se diminuído significativamente no grupo 4 quando comparado com os demais grupos. Já o colesterol LDL estava diminuído significativamente quando comparado com o grupo 2. O colesterol VLDL está adequado nos grupos. Os quatro grupos apresentaram concentrações de triglicerídeos elevadas, porém o grupo 1 apresentou aumento significativo quando comparado com os demais grupos. As concentrações de Alumínio encontram-se aumentadas significativamente no grupo 1 quando comparado com os demais grupos (Tabela 3). 
Tabela 3. Resultados dos exames anuais.

\begin{tabular}{lccccc}
\hline & Grupo 1 & Grupo 2 & Grupo 3 & Grupo 4 & p \\
\hline HDL $(\mathrm{mg} / \mathrm{dL})$ & $49,91 \pm 13,32^{*}$ & $49,50 \pm 16,60^{*}$ & $49,75 \pm 19,32^{*}$ & $37,25 \pm 21,98^{*}$ & 0,05 \\
\hline LDL $(\mathrm{mg} / \mathrm{dL})$ & $80,54 \pm 36,97$ & $101,3 \pm 19,21^{*}$ & $91,43 \pm 35,13$ & $76,73 \pm 40,05^{*}$ & 0,05 \\
\hline VLDL $(\mathrm{mg} / \mathrm{dL})$ & $39,72 \pm 21,75$ & $33,50 \pm 11,82$ & $43,95 \pm 20,74$ & $46,27 \pm 32,69$ & 0,05 \\
\hline TG $(\mathrm{mg} / \mathrm{dL})$ & $244,1 \pm 246,7^{*}$ & $162,9 \pm 57,87^{*}$ & $215,1 \pm 103,2^{*}$ & $233,1 \pm 162,9^{*}$ & 0,05 \\
\hline Alumínio (g/L) & $34,66 \pm 43,88^{*},{ }^{\#}$ & $31,34 \pm 30,56^{*}$ & $15,34 \pm 7,943^{*},{ }^{*}$ & $22,51 \pm 13,68^{*}$, & 0,05 \\
\hline
\end{tabular}

HB - hemoglobina, HT - hematócrito, TGP - transaminase glutâmico pirúvica, CR - creatinina urinária, PCR - proteína C reativa, PTH - paratormônio. Valores expressos em Média $\pm \mathrm{DP},{ }^{*}$ ou ${ }^{\#}$ diferença significativa em comparação ao Grupo sem sinal.

Em relação à equipe de enfermagem nota-se que o enfermeiro tem um papel muito importante no cuidado do paciente que realiza procedimentos de HD é ele quem auxilia nas dificuldades enfrentadas pelos pacientes, quem contribui para a adaptação das novas rotinas impostas pelo tratamento, ajudando esses pacientes a enfrentarem as dificuldades do dia-adia e se sentirem capazes e responsáveis de realizarem $\mathrm{o}$ autocuidado mesmo com as limitações que a doença e a HD causam.

\section{DISCUSSÃO}

Nosso estudo encontrou um perfil predominante de pacientes em idade adulta (56\%), entre 19-64 anos, e discreta predominância do sexo masculino. Estando em concordância com o cenário nacional exposto no Censo Brasileiro de Diálise Crônica de 2012 da Sociedade Brasileira de Nefrologia, assim como é compatível com os resultados de diversos estudos, sendo levantadas duas hipóteses, a primeira é de que o gênero masculino pode ser um fator de risco não modificável para o desenvolvimento de DRC e a segunda, que possivelmente, o número ligeiramente superior de homens com DRC, deva-se ao fato de que, geralmente, as mulheres se preocupam mais com a própria saúde, seguindo recomendações sobre cuidados necessários de forma mais rígida prorrogando, assim, o tempo para o surgimento da DRC em seu estado terminal ${ }^{11-13}$.

A grande maioria dos pacientes de nosso estudo apresentou, após análise dos parâmetros bioquímicos e hematológicos, alterações nas concentrações de PCR, HB, HT, ureia pré-diálise, PCR, Transferrina e Ferritina, sendo observada diferença significativa, em vários desses parâmetros, entre o grupo 1 (3-7 meses em HD) em relação aos demais grupos (8-12, 13-24 e 2536 meses em HD).
Encontramos em todos os pacientes níveis de PCR acima de $8 \mathrm{mg} / \mathrm{L}$, geralmente concentrações elevadas estão relacionados a um estado inflamatório, o qual pode ser oriundo do próprio procedimento hemodialítico ou das complicações relacionadas à uremia, como redução da depuração renal das citocinas, estresse oxidativo, acúmulo de produtos finais da glicação avançada (AGE) e redução da resposta imune em razão da ação prejudicada de neutrófilos e de células T. Fatores esses que contribuem de forma significativa para a inflamação subclínica existente nos pacientes com DRC ${ }^{5}$.

Essa inflamação, frequentemente vista em pacientes que realizam hemodiálise, pode também contribuir para o desenvolvimento da anemia através da eritropoiese e consequente deficiência de eritropoietina ${ }^{14}$. Contudo, existem várias outras causas para a anemia nesses pacientes que são de extrema importância, entre os quais a falta de ácido fólico e vitamina B12, hemorragia gastrintestinal e deficiência de ferro. Porém, se não houver a existência de quaisquer outras causas para a anemia, a deficiência de eritropoietina deve ser considerada como a causa subjacente, devendo ser iniciado o tratamento com agentes estimuladores da eritropoiese ${ }^{15}$.

A anemia derivada da DRC é normocítica e normocrômica, sendo atribuída a um déficit relativo de eritropoietina. A definição de anemia não é ainda uma questão resolvida. Porém seu diagnóstico, em pacientes em hemodiálise, é realizado quando os níveis de $\mathrm{HB}$ encontram-se menores que 11 a $12 \mathrm{~g} / \mathrm{dL}$ e os valores de HT abaixo de $33 \%$ de acordo com o Guia Guidelines of National Kidney Foundation Kidney Disease Outcome Quality Initiative (NKF-KDOQI) ${ }^{15,16}$.

Nosso estudo identificou, em todos os grupos, concentrações de HB e HT depletados. Apesar de não ter ocorrido diferença significativa 
entre os grupos, verificamos que ocorreu diminuição nas concentrações de $\mathrm{HB}$ e no $H T$ independe do tempo que o indivíduo realiza HD, e que, mesmo com a reposição continua de eritropoietina recombinante humana durante as sessões de HD, estes pacientes ainda apresentam um quadro anêmico, associando-se desfavoravelmente à evolução do paciente em diversas doenças, demonstrando maior percentual de internações, maior permanência hospitalar e um número maior de comorbidades $^{16,17}$.

Embora os pacientes do nosso estudo tenham apresentado quadro anêmico, as concentrações de ferro sérico e albumina encontraram-se adequados. Tal fato justifica-se, possivelmente, pelos baixos teores de HB dos reticulócitos estar mais associada com inflamação, medida pela PCR, do que com a própria deficiência de ferro ${ }^{17}$. Foram identificados também, concentrações elevadas de ferritina e reduzidos de transferrina. A ferritina no soro é um marcador de reservas de ferro no fígado e no sistema reticulo endotelial, bem como uma proteína de fase aguda, encontrando-se, portanto, elevada na presença de inflamação, resultando em uma baixa saturação de transferrina ${ }^{13,14}$.

Pacientes com DRC também apresentam prejudicadas a excreção de solutos não voláteis tóxicos, com consequente aumento das concentrações plasmáticas de todos os catabólitos derivados principalmente de metabolismo de proteínas, caracterizada pelo aumento da ureia e CR. A dosagem de CR é utilizada no diagnóstico da função renal, sendo um indicador mais sensível e especifico seguido da dosagem de ureia. Ambos auxiliam na monitoração, análise e prognóstico da progressão da doença renal ${ }^{13}$.

Em relação à função renal, todos os grupos apresentaram concentrações aumentadas de $C R$ e ureia pré-diálise dentro do período de estudo, estando em concordância com outro estudo $^{13}$. Apesar das concentrações elevadas no momento de pré, os valores de ureia foram reduzidos em todos os pacientes, atingindo os valores de referência, após a sessão, demonstrando que a hemodiálise cumpriu a sua função.

Observamos que o grupo com menos tempo de HD (Grupo 1) manteve normais suas concentrações de potássio, enquanto os grupos mais antigos encontravam-se com as concentrações de $\mathrm{K}^{+}$significativamente elevados. Essa diferença pode ser justificada devido à capacidade de excreção de potássio estar relativamente bem preservada na insuficiência renal crônica, porém à medida que a doença avança para a fase terminal, essa capacidade de excreção fica comprometida. Isto se deve a um aumento na excreção de $K^{+}$por néfron ${ }^{18}$.

Nosso estudo observou em todos os grupos altas concentrações de fosfato, já que na $\mathrm{DRC}$, a perda progressiva de néfrons funcionais induz a retenção de fosfato, que ao atingir a fase terminal manifesta-se com o aparecimento da hiperfosfatemia. A hiperfosfatemia, fósforo acima de 2,1 $\mathrm{mmol} / \mathrm{L}$, é uma característica comum da DRC, evidenciada na maioria dos indivíduos que realizam HD, e está relacionada a aumento da morbidade e mortalidade por doenças cardiovasculares, em comparação com pacientes que apresentam concentrações de fósforo entre 0,7 e $2,1 \mathrm{mmol} / \mathrm{L}^{19}$.

Complicações ateroscleróticas de artérias coronárias e cerebrais são importantes e frequentes complicações em pacientes submetidos à HD crônica ${ }^{17}$. Nosso estudo encontrou concentrações de colesterol HDL minimamente reduzido nos grupos 1 e 4, e adequados nos grupos 2 e 3 . O colesterol LDL encontra-se adequado para todos os grupos, porém com pequena elevação no grupo 2. O colesterol VLDL está adequado nos grupos 1 e 2 e elevado nos grupos 3 e 4 . Os quatro grupos encontram-se com as concentrações de triglicerídeos elevadas. Porém, nenhuma dessas variáveis obteve significância estatística. Importante ressaltar que, nem colesterol total nem LDL estão positivamente associados com a mortalidade, pois concentrações reduzidas de LDL e colesterol total estão associados a maiores riscos cardiovasculares. Portanto, um paciente que realiza $\mathrm{HD}$ e tem baixa concentração de LDL tem mais um motivo para alarme do que para satisfação ${ }^{18}$.

Os resultados dos nossos estudos estão em concordância com pesquisas anteriores. Os achados podem ser explicados devido à própria evolução da DRC para seu estado terminal, a qual gera nos indivíduos um estado inflamatório crônico, desencadeando disfunções endoteliais, indução e aceleração do processo de aterosclerose e arteriosclerose, alteração do estado nutricional, anemia e disfunção imune tornando-os mais susceptíveis a infecções e reações adversas ao processo de HD. Por tais 
razões, são inúmeras as tentativas de reduzir a exposição destes pacientes a produtos bacterianos durante o processo hemodialítico, melhorando a qualidade dos sistemas de água $^{17,18}$.

Estudos apontam que apesar da HD ser uma estratégia indispensável para pacientes com DRC avançada, a grande quantidade de água que entra em contato com 0 paciente pode desencadear reações indesejáveis se as características físico-químicas ou microbiológicas não estiverem de acordo com os níveis permitidos.

Ao analisar alguns parâmetros hematológicos e bioquímicos, verificamos que todos os pacientes em tratamento hemodialítico desenvolveram diversas alterações em decorrência da DRC e das injúrias consequentes ao processo de HD. Evidenciando-se a presença de anemia, com concentrações reduzidas de hemoglobina e hematócrito, e de alterações em tantos outros parâmetros que direta ou indiretamente geram um aumento crônico do quadro inflamatório e comprometimento imunológico. Para tanto, ressalta-se a necessidade de um monitoramento rigoroso dos parâmetros bioquímicos e hematológicos apresentados pelos pacientes e dos parâmetros que asseguram a qualidade da água utilizada no processo de HD, já que a mesma é um fator independente de biocompatibilidade, e contribui de forma impar para redução da inflamação e correção da anemia ${ }^{9,21}$.

Sabe-se, no entanto, que correlacionar tais fatores é um processo difícil, seja pelos possíveis viesses ou pelas barreiras impostas pelos próprios serviços de HD perante a realização deste tipo de pesquisa. No entanto, tais barreiras devem ser superadas, para que se possa identificar fatores frágeis e buscar um tratamento ideal, através da conscientização dos serviços de HD de que novas pesquisas do gênero devem ser realizadas e aprofundadas com o intuito de melhorar os desfechos de pacientes com doença renal em estágio terminal.

Visto que a equipe de enfermagem assim como em inúmeros serviços do ramo se faz indispensável, essencial e decisiva, são parte da influência aos pacientes para continuarem o tratamento, esclarecendo dúvidas sobre mudanças corporais, acessos vasculares, fístula, dieta, uso de medicações, orientações sobre a permanência na máquina durante as sessões de $\mathrm{HD}$ e sempre incentivando-os ${ }^{11,22}$.
A equipe de enfermagem por estar mais frequentemente em contato com os pacientes em HD desempenha papel de ouvinte, possibilitando a verbalização das frustrações, decepções e barreiras que a DRC impõe $e^{11,12}$.

\section{CONCLUSÃO}

Ao analisar alguns parâmetros hematológicos e bioquímicos, verificamos que todos os pacientes em tratamento hemodialítico desenvolveram diversas alterações em decorrência da DRC e das injúrias consequentes ao processo de HD, evidenciando a presença de anemia, com concentrações reduzidas de HB e HT, e de alterações em tantos outros parâmetros que direta ou indiretamente geram aumento crônico do quadro inflamatório e comprometimento imunológico. Nessa vertente, ressalta-se a importância da atuação da equipe de enfermagem com os pacientes, é necessário que os profissionais não tenham somente a fundamentação cientifica e competência técnica, mas que possam estabelecer maior diálogo com os seus pacientes resultando na compreensão de suas necessidades e também ressalta-se a importância de novas pesquisas que correlacionem os parâmetros bioquímicos e hematológicos apresentados pelos pacientes evitando ou descobrindo precocemente possíveis complicações.

\section{CONFLITO DE INTERESSE}

Os autores declaram não haver qualquer potencial conflito de interesse que possa interferir na imparcialidade deste trabalho científico.

\section{REFERÊNCIAS}

1. BRASIL. ANVISA. Agência Nacional de Vigilância Sanitária. Gestão 2005-2010: principais realizações / Agência Nacional de Vigilância Sanitária. Brasília: ANVISA, 2010.

2. Nunes $A$. O envelhecimento populacional e as despesas do Sistema Único de Saúde. In: Camarano AA (Organizadora. Os novos idosos brasileiros - Muito além dos 60 . Rio de Janeiro: IPEA; 2004.

3. Ribeiro AG, Cotta RMM, Ribeiro SMR. A Promoção da Saúde e a Prevenção Integrada dos Fatores de Risco para Doenças Cardiovasculares. Ciên Saúde Col. 2012;17(1):7-17. https://doi.org/10.1590/S1413$\underline{81232012000100002}$ 
4. Braga FLM, Arruda IG, Diniz AS, Cabral PC, Lemos MCC, Braga MDM, Chaves Jr HC. Disfunção renal e marcadores inflamatórios em hipertensos atendidos em hospital universitário. Arq Bras Cardiol. 2013;100(6):538-45. DOI: http://dx.doi.org/10.5935/abc.20130102

5. Barbosa ACSCS, Salomon ALR. Resposta inflamatória de pacientes com doença renal crônica em fase pré-dialítica e sua relação com a ingestão protéica. Com Ciên Saúde. 2012;24(12):11-25.

6. Bastos MG, Bregman R, Kirsztajn GM. Doença renal crônica: frequente e grave, mas também prevenível e tratável. Rev Assoc Med Bras. 2010;56(2):248-53.

DOI:

http://dx.doi.org/10.1590/S0104-

\section{8}

7. Romão Jr JE. Doença Renal Crônica: epidemiologia e classificação. J Bras Nefrol. 2004;26(3 Supl 1): 1-3.

8. Silva LK, Bregman R, Lessi D, Leimann B, Alves $M B$. et al. Ensaio sobre a cegueira: mortalidade de pacientes com doença renal crônica em hemodiálise de emergência. Ciên Saúde Col. 2012;27(11):2971-80.

https://doi.org/10.1590/S1413-

81232012001100014

9. Akhmouch I, Asserraji M, Bahadi A, Bouaiti E, Alayoude $M$, Aatif $T$. et al. Effect of the quality of water used for dialysis on the efficacy of hemodialysis: A single-center experience from Marocco. Saudi J Kidney Dis Transpl. 2011;22:576-80.

10. Cesarino CB, Casagrande L.D.R. Paciente com insuficiência renal crônica em tratamento hemodialtico: atividade educativa do enfermeiro. Rev Latino-Am Enferm. 1998;6(4):31-40. DOI: https://doi.org/10.1590/S0104-

\section{5}

11. Ferreira AFA. O papel do enfermeiro na assistência de enfermagem ao paciente em tratamento hemodialítico. 2014. 24p. [Monografia]. Curso de Pós-Graduado em Nefrologia do Centro de Capacitação Educacional, Instituto Nacional de Ensino e Pesquisa - INESP, Recife, 2014.

12. Egbi OG, Okafor UH, Miebodei KE, Kasia $\mathrm{BE}$, Kunle-Olowu OE, Unuigbe El. Prevalence and correlates of chronic kidney disease among civil servants in Bayelsa state, Nigeria. Niger J Clin Pract. 2014;17:602-7. DOI: https://doi.org/10.4103/1119-3077.141426

13. Peres LAB, Biela R, Herrmann M, Matsuo $T$, Ann HK, Camargo MTA. Estudo epidemiológico da doença renal crônica terminal no oeste do
Paraná: uma experiência de 878 casos atendidos em 25 anos. J Bras Nefrol. 2010;32(1):51-6. DOI: https://doi.org/10.1590/S0101-

\section{0}

14. Bueno CS, Frizzo MN. Anemia na doença renal crônica em hospital da região noroeste do estado do Rio Grande do Sul. J Bras Nefrol. 2014;36(3): 304-14.

15. Sany D, Elsawy AE, Elshahawy Y. Hepcidin and regulation homeostasis in maintenance hemodialysis patients. Saudi J Kidney Dis Transpl. 2014;25:967-73.

DOI:

https://doi.org/10.4103/1319-2442.139868

16. Javidan AN, Shahbazian H, Emami A, Yekanjnejad MS, Razavi HE, Farhadkhani M. Safety and efficacy of PDpoetin for management of anemia in patients with end stage renal disease on maintenance hemodialysis: results from a phase IV clinical trial. Hematol Rep. 2014;6:5195.

17. Alves MAR, Gordan PA. Diagnóstico de anemia em pacientes portadores de doença renal crônica. J Bras Nefrol. 2014;36(Supl. 1):9-12.

18. Kaysen GA. Biochemistry and biomarkers of inflamed patients: why look, what to assess. Clin J Am Soc Nephrol. 2009;4:S56-S63. DOI: https://doi.org/10.2215/CJN.03090509

19. Riella MC. Princípios de nefrologia e distúrbio hidroeletrolítico. 4.ed. Rio de Janeiro: Guanabara Koogan; 2003.

20. Gross P, Six I, Kamel S, Massy ZA. Vascular Toxicity of Phosphate in Chronic Kidney DiseaseBeyond Vascular Calcification. Circul J. 2014;78(10):1-8.

DOI:

http://dx.doi.org/10.1253/circi.CJ-14-0735

21. Wang $S$, Qin $L, W u T$, Deng B, Sun $Y$, Hu D. Elevated cardiac markers in chronic kidney disease as a consequence of hyperphosphatemiainduced cardiac myocyte injury. Med Sci Monit. 2014;20:2043-53.

DOI:

\section{https://doi.org/10.12659/MSM.890909}

22. Rezende RC, Porto IS. Cuidado de enfermagem para clientela em hemodiálise: suas dimensões instrumentais e expressivas. Rev Eletr Enf. 2009;11(2):266-74. DOI: https://doi.org/10.5216/ree.v11.46939

Recebido para publicação em 30/06/2016

Revisado em 30/06/2017

Aceito em 08/02/2018 